\title{
The logistics network digital twin in view of concept of the non-destructive quality control methods
}

\author{
Irina Kapustina ${ }^{1}$, Olga Kalinina $^{1}$, Alina Ovchinnikova ${ }^{1}$, Sergei Barykin ${ }^{1 *}$ \\ 1, Peter the Great St. Petersburg Polytechnic University, Polytechnicheskaya, 29, St. Petersburg, \\ 195251, Russian Federation
}

\begin{abstract}
The purpose of the research consists in improving the theoretical basis for non-destructive quality control methods in the context of digitalization and an active transition to Industry 4.0. The methodology used by authors include the consideration of the logistics network digital twin, as well as globalization of markets and ever-increasing global competition in all areas. The article discusses non-destructive quality control methods based on the process of modeling digital twins and the authors' approach proves the advantages of just such an innovative concept of expanding the definition of logistics network digital twin in comparison with the traditional methods of non-destructive quality control. It could be shown that this approach eventually leads the ability of industries to switch to digitalization of their processes. Saying precisely the improving of the quality control methods could be treated as digitalization of their supply chains, reflecting the entire product life cycle. We have displayed a digital supply chain, which includes the method of non-destructive testing, like a digital twin. The main difference between the digital supply chain and the traditional supply chain is noted. The ideas of large audit companies such as Gartner's, Siemens and Deloitte are analyzed. What are the benefits of using a digital twin as a nondestructive testing method? All participants of this digital supply chain receive their benefits in terms of digitalization and market competition.
\end{abstract}

\section{Introduction}

The relevance of this study is that now industry is committed to innovation and to the acclaimed Industry 4.0. Markets are globalizing and global competition in all areas is constantly increasing. And in order to be a competitive enterprise, it is necessary to develop and have advantages that are just expressed in the ability of industries to switch to digitalization of their processes.

Large audit companies such as Gartner's [1], who writes about the role of the digital supply chain in the digital era, are now writing about digitalization, but he does not consider it throughout the entire product life cycle. Siemens [2] considers the benefits of digital transformation and the issue of optimizing the individual steps of the value chain. Deloitte

\footnotetext{
* Corresponding author: sbe@ @list.ru
} 
$[3,4]$ describes the digital twin as a digital trace of a product throughout its entire life cycle. One of the largest KPMG networks in the world [5] also analyzes production readiness for the digitalization era. But no one specifically raised the stage of quality control in the discussion of the digital supply chain, for which a digital twin is needed.

It's also not a secret for anyone that company managers strive to extract as much profit from production as possible, and one way to increase profit is to reduce costs. One of these methods will be considered in the article.

\section{Materials and methods}

In order to select the necessary material for the study, a special scheme of scientific research was developed (Fig. 1).

Through which there was a filtration of various sources. So, all literature was eventually divided into methodological support and theoretical positions. Basically, no one used the concept of a digital twin as a method of non-destructive testing, which introduces novelty into our article.

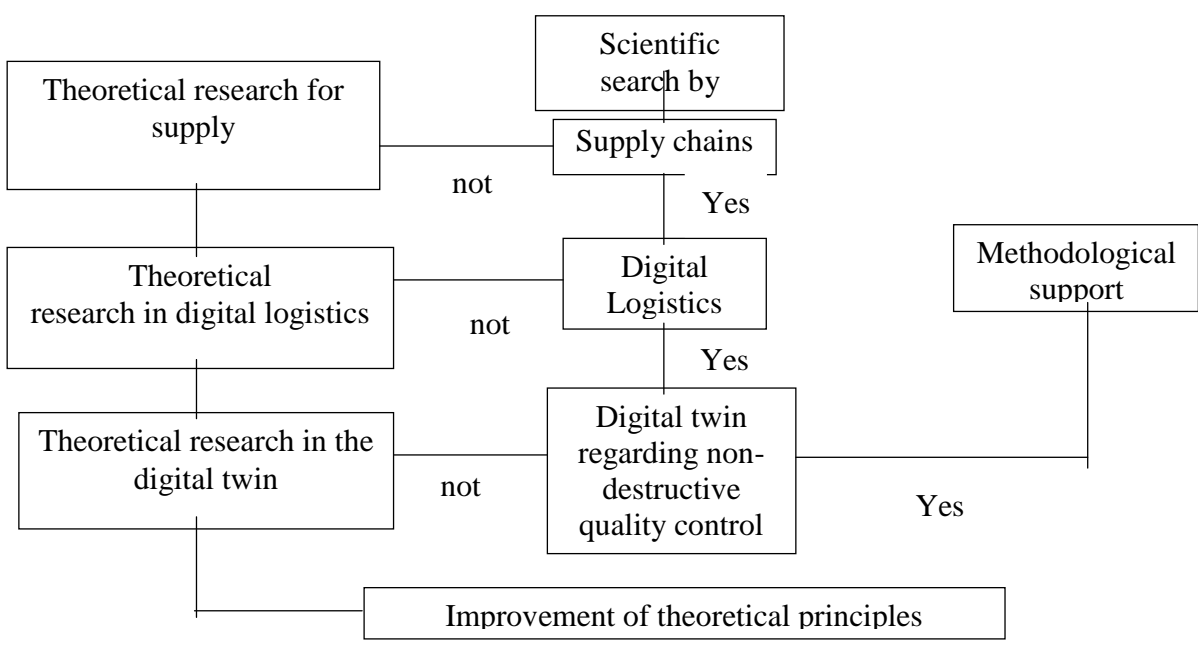

Fig. 1. Scheme of scientific search by sources.

The main definitions used for the study:

Non-destructive testing (NDT) - includes a wide group of techniques utilized in science and industry to evaluate the properties of a material (such as residual stresses) without causing any major damage. [6] A digital twin - an evolving digital profile of the historical and current behavior of a physical object or process that helps optimize business performance. [7] A digital twin - is an accurate virtual model of a product or manufacturing enterprise. [2] Digital supply chains - represent a dramatic shift from linear, sequential supply chain operations to an interconnected, open ecosystem. [3]

\section{Results}

Now manufacturers spend a lot of resources when conducting control of the resulting product. Often these are destructive-type controls, when a certain number of test objects is taken from 
a batch of products and all kinds of tests are carried out with them to determine the required indicators required by the customer. When the tests are completed, the selected batch of products is no longer suitable for further use. In this case, the manufacturer incurs losses due to the production of additional copies, which the customer, of course, does not pay, and also due to the further need for disposal of samples.

Therefore, it is much more profitable for the manufacturer to resort to non-destructive testing methods.

Non-destructive testing (NDT) - includes a wide group of techniques utilized in science and industry to evaluate the properties of a material (such as residual stresses) without causing any major damage. [6]

Benefits:

1. There is no direct physical contact with the test sample, as a result of which it may lose its function. This is very important when testing expensive samples.

2. A minimum of preparation for the study and evaluation of the characteristics of the object.

3. It is possible to observe dynamics in the entire product life cycle. The ability to find out the cause of failure and eliminate the root cause.

4. It is not necessary to suspend the work of the test object, since research does not affect the properties and functions of the object.

5. The sample can be tested using several non-destructive testing methods to obtain several options, since during the test its physical integrity is not violated.

6. No need to produce additional probes for testing, the test sample will not lose the ability to perform its assigned functions in production.

Disadvantages:

1. The condition of objects is determined visually in most cases, only occasionally do special devices are used for registration.

2. It is impossible to exclude the influence of external factors that may affect the results of the study, but which have no relation to the environment of use of the sample.

3. It is necessary to create real working conditions for the test sample in order to obtain accurate control data.

There is a standard that classifies the types and methods of non-destructive testing. Nondestructive testing, in accordance with the physical phenomena underlying it, is divided into types:

1. Acoustic;

2. Eddy current;

3. Magnetic;

4. Optical;

5. Penetrating substances;

6. Radiation;

7. Radio wave;

8. Thermal;

9. Electrical.

Each presented non-destructive testing method has its undeniable advantages. However, with the constant development of the world around us, problems such as globalization of markets, ever-increasing global competition in all areas, the use of high-tech innovations, the emergence of highly complex scientific and technical problems that require Russian industry to develop innovatively, reduce development time, low prices and with high quality products.

To meet these requirements, the world is undergoing a global digital transformation of the economy into a digital economy, and the high-tech industry into a digital industry.

The new technology of non-destructive testing includes the modeling method. Modeling - allows the study of physically real objects on their models. This can be a study of both an 
object and processes or phenomena in order to obtain an answer about the origin of these phenomena and for their future prediction.

The world around us is changing daily with the development of human civilization. Therefore, the technical and technological development of production is changing. This led to the concept of Industry 4.0, a model that shows how industrial production follows the latest developments and changes over time. Thus, man, machine, and production itself constitute power in one intelligent and independent network. [8]

The use of digital technology is leading to radical changes in business models. To achieve these goals, the so-called digital innovation is needed. In order to bring to life many innovations as quickly as possible, production must become more flexible. Two factors that will help achieve this goal are hardware and software solutions for real-time data evaluation. [8]

One of the key technologies is the digital twin. It can successfully act as a substitute for expensive field monitoring methods, while it does not cause any damage to the physical object. A digital twin regards to an evolving digital profile of the historical and current behavior of a physical object or process that helps optimize business performance. [7]

A digital twin is an accurate virtual model of a product or manufacturing enterprise. It reflects their development throughout the life cycle and allows operators to predict behavior, optimize performance and implement ideas from previous experience in design and production. [2]

This technology makes it possible to simulate a variety of situations that arise during the operation of the investigated object. Thus, the digital twin allows you to draw up a plan for technological processes, avoiding unforeseen situations.

To create a comprehensive model of the digital twin, numerical methods are used to model various physical processes in the materials of the object. This helps to understand how the product responds to certain operational loads.

The industry uses such systems as CAD models (computer aided design tools), they contain information about the appearance and parameters, structure and materials of the object, as well as processes and other characteristics. And there is also an FMEA-model (analysis of the types and consequences of failures) that allows you to evaluate the reliability of the system by analyzing the failure and identifying the most critical moments in production. Through the use of modeling, it has become possible to avoid increasing costs throughout the entire life cycle, due to the fact that you do not have to make the necessary changes to the product at the very last moment. The simulated product has already calculated all possible changes in advance. So the most expensive stage in production is designing based on the simulation of a digital twin. And due to the fact that all the focus is on design, production significantly minimizes its costs and expenses, and it is possible to develop new high-tech products in less time.

Quality control using digital technology is gradually gaining popularity in Russian production. According to a study by KPMG (one of the largest professional service networks in the world and one of the Big Four audit companies) in Russia, which was attended by over 100 of the largest Russian companies, large companies have already embarked on the path of digital transformation. However, most of them do not yet have a comprehensive digitalization program. [5]

The main obstacles that Russian companies face on the road to digitalization are presented in the figure taken from KPMG research (Fig. 2). 63\% of Russian companies said they have a well-developed digital transformation program. Also, the leaders of $43 \%$ of Russian organizations prefer the introduction of only established themselves in the technology market. $88 \%$ of executives vote for the development of innovative technologies opens up more opportunities than threats. A new CDO position (Chief Digital Officer, chief digital director) was introduced, which so far exists only in $8 \%$ of the companies surveyed. [5] 


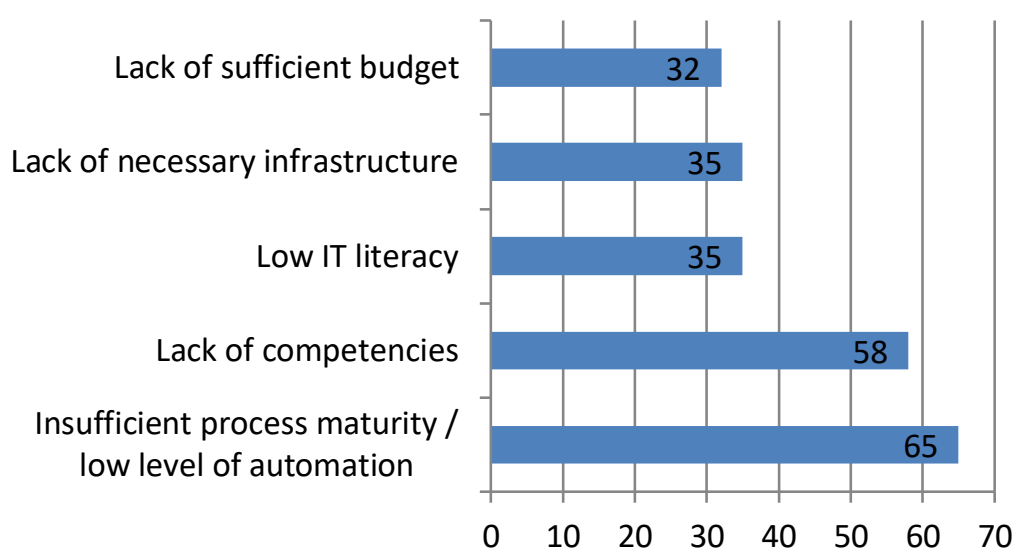

Fig. 2. Obstacles to digitalization.

The study also analyzed what effects Russian companies expect from the introduction of innovative digital technologies, they are presented in the figure taken from KPMG studies (Fig. 3):

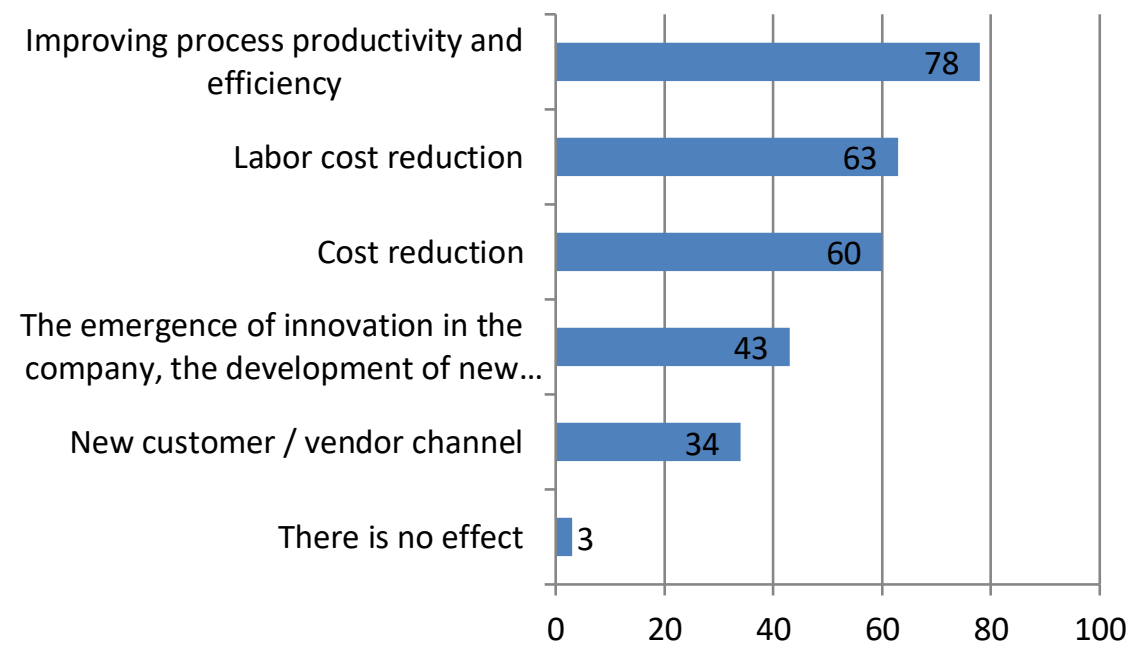

Figure 3. The effect of implementing the digital technology.

The goal of all these measures is to increase productivity (due to a sharp reduction in the time period between the development of a new product and its delivery to consumers by $50 \%$ ), efficiency (automation allows greater flexibility, better product quality and more efficient production) and energy saving (for example, pending processing of materials, robots can be turned off, if necessary, which saves up to 15 electricity) to ensure competitiveness in the global market. [8]

Also, industrialists receive resource savings and reduce the time required for the design and testing of products. The corporations have calculated that in some areas, virtual tests can halve the number of full-scale, to abandon the manufacture of dozens of trial models.

Since Russian manufactures are already ready and open for new digital technologies and for a complete change in their entire business model, they clearly understand that a digital twin can be not only a product model. 
The comprehensive concept of the digital twin consists of three forms: the digital twin of the product, the digital twin of production and the effectiveness of the digital twin of both product and production (Fig. 4). [2]

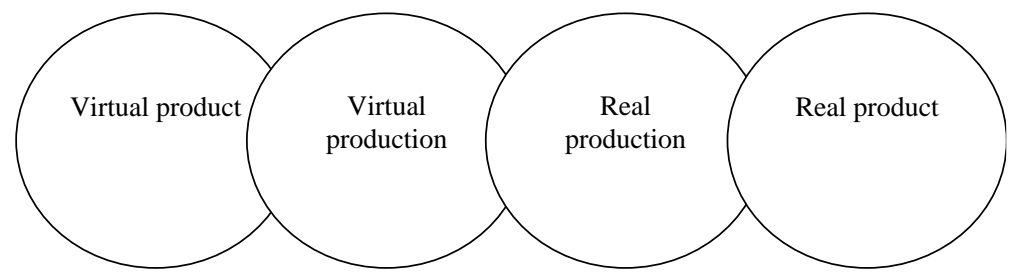

Fig. 4. Comprehensive digital twin concept.

The digital twin concept includes product design as well as virtual, system-oriented product development. This allows you to design, simulate and validate complex products, simulate multiphysics, automate electronic design and manage software - in different areas without the need for physical prototypes.

Digital twin concept can be defined as follows. Digital planning, modeling and production optimization with automatic PLC code generation (programmable logic controller) creates a digital production twin. This definition is included in Factory of the Future used to call such a production in the era of Industry 4.0.

The effectiveness of a digital twin involves a combination of the results of real production and product. In the real world, the digital performance twin constantly receives data from the product and production facilities, which leads to new conclusions. By connecting with integrated automation components, the workshop provides all the necessary data. The transfer of all information along the entire value chain through MindSphere (a cloud-based operating system that allows you to connect any physical device to a digital information space) - up to the product design - creates a completely closed decision-making cycle for continuous optimization of production and product in the real world.

The ultimate goal of the digital twin is a closed loop of communication between the virtual world of product development and production planning with the physical world of the production system and product performance. Thanks to this connection, practical information can be obtained from the physical world for making informed decisions at the stages of the entire life cycle of products and production operations. [2]

The implementation of digital twinning technologies is a rather serious project, which may require large investments and preliminary estimates of their payback. However, according to a Deloitte study, this technology is rapidly spreading in various industries, including aerospace, retail, healthcare, and other sectors. Digital counterparts accelerate the development of products and processes, optimize the execution of work and help in preventive. [4] According to the report, the global market for digital twin technology will reach $\$ 16$ billion by 2023 .

As previously noted, a digital twin can be used at all stages of a product's life, creating reports on its work in real time. And besides the manufacturer, customers also want to use this data. And here we are faced with the supply chain and the need for its transparency.

According to Gartner: Supply chain visibility SCV is a competency that will soon become standard business practice. SCV is a timely, accurate and complete presentation of plans, events and data throughout the supply chain, including external partners. [1]

A digital twin that performs the task of non-destructive testing will occupy an important place in the chain from the supplier to the customer. So innovative digital technologies will allow companies to collect high-quality relevant data that they can analyze, integrate and interpret. Automation, forecasting, and robotics will be built on this data - all the innovations 
that will replace the traditional supply chain management.

Conventional supply chains with the transition to Industry 4.0 are also gaining new development, already as digital supply chains. Digital supply chains should cover all components of the supply chain, but implementation can begin without disrupting existing processes. Companies integrating digital technology in their supply chain can quickly increase service levels and reduce costs by up to $30 \%$. Equally important are the opportunities that digital technologies provide for developing new business models and new strategies. [9]

Determining the ideal future state for the supply chain allows leadership teams to identify missing opportunities and begin to create them. One of the tasks for the leadership groups planning to modernize the supply chain is to identify the next steps that will help to recoup future innovations. Successful companies are building a short-term roadmap with concrete initiatives that will quickly start to be profitable and provide the flexibility to achieve longterm supply chain goals. [9]

Digital supply chains, in turn, meet the current trend of business development and digital technologies, thanks to which companies have the opportunity to gain new competitive advantages (cost reduction, efficiency, and time reduction). The use of such solutions makes it possible to move to a new level of cooperation and create transparency in the entire supply chain. This creates the basis on which modern logistics technologies and strategies can be implemented and additional benefits are created for the effective management and development of supply chains. Supply chain management (SCM) - is an external service that provides standardized processes through a cloud-based technology platform. [1]

Effective exchange of information is the main key to the success of any supply chain in our time. The traditional supply chain is faced with problems and operational errors caused by incomplete and untimely information. The possibility of failure is great, such as a sudden shift in market demand, a shortage of necessary raw materials, and a change in external factors. The manufacturer cannot influence the given failures in any way, but by receiving timely information, it is possible to prevent losses or change something.

Therefore, the main goal of the digital supply chain is the transparency of both supply and production for all interested parties.

The virtual method of non-destructive testing, namely the digital twin of the product, successfully occupies a place in the digital supply chain and participates in the information flow (Fig. 5).

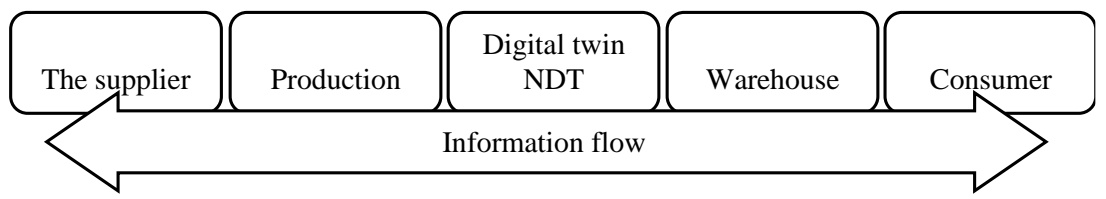

Fig. 5. Digital supply chain.

Such technologies allow all interested parties, at least somehow related to the supply chain, to seamlessly interact with each other in such matters as the development, production, storage, delivery and subsequent necessary maintenance of the product.

Digital counterparts have become a really strong catalyst for the development of Russian companies. Through the use of these technologies, technical support of systems is simplified, various resources are saved, the risks of errors and failures are minimized and predicted throughout the entire product life cycle from design to the very end of the customer's operation, which extends the period of stable operation of the product at the customer due to open information. All this allows the business to grow, receive maximum return on investment, gain new competitive advantages and build loyalty. 


\section{Discussion}

In this study, we would like to pay more attention to all stages of the product life cycle in digital supply chains. Consider all the modern technical capabilities of Russian industries and their ability to transform their business model. I would also like to talk about already experienced foreign production. But we have outlined the outline of the object of study of the digital twin, as a tool for non-destructive testing in the digital supply chain.

\section{Conclusions}

In this study, we succeeded in linking such an introduction as modeling a digital twin with the subsequent creation of transparent supply chains, and to prove how one change can turn ordinary production into digital. The advantages that are acquired by the production following innovative digital technologies were also shown. KPMG research results in Russia were analyzed in relation to the readiness of Russian enterprises for digital transformation. And a positive attitude of $88 \%$ of participants towards digitalization was revealed. As a result, focusing on improving processes, we get cost reduction, increasing efficiency and competitiveness. Anyway, anticipating change is a strategic leap into the future.

\section{References}

1. Gartner's Hype Cycle Reveals the Digitalization of the Supply Chain (2017) https://www.gartner.com/en/newsroom/press-releases/2017-09-11-gartner-hype-cyclereveals-the-digitalization-of-the-suppl-chain

2. Siemens. Realize your digital transformation now. The Digital Enterprise Suite for Product Manufacturers, 22 (2019) https://new.siemens.com/global/en/company/topicareas/digital-enterprise/discrete-industry.html

3. Deloitte. Digital supply networks https://www2.deloitte.com/us/en/pages/deloitte-analytics/articles/digital-supplynetworks.html

4. Deloitte University Press. Industry 4.0 and the digital twin, 20 (2017) https://www2.deloitte.com/content/dam/Deloitte/cn/Documents/cip/deloitte-cn-cipindustry-4-0-digital-twin-technology-en-171215.pdf

5. KPMG. Digital technologies in Russian companies. The results of the study, 40 (2019) https://assets.kpmg/content/dam/kpmg/en/pdf/2019/01/en-ru-digital-technologies-inrussian-companies.pdf

6. M.M. Shokrieh, A.R. Ghanei Mohammadi. Residual Stresses in Composite Materials, 58-75 (2014) https://doi.org/10.1533/9780857098597.1.58

7. D. Kokorev, A. Yurin. Digital twins: concept, types and benefits for business, 31-35 (2019) https://doi.org/10.24411/2520-6990-2019-10264

8. D. Vuksanović, J. Ugarak, D. Korčok. Sinteza 2016 - International Scientific Conference on ICT and E-Business Related Research, 293-298 (2016) http://doi.org/10.15308/Sinteza-2016-293-298

9. S. Israelit, P. Hanbury, R. Mayo and T. Kwasniok. Build a Digital Supply Chain That Is Fit for the Future. Digital tools can help companies stay a step ahead of changing industries. https://www.bain.com/insights/build-a-digital-supply-chain-that-is-fit-forthe-future/ 